\title{
Éditorial. Méthodologies de recherche et coopération internationale en éducation
}

\author{
Barbara Class, Université de Genève \\ Thibaut Lauwerier, Université de Genève
}

\section{DOI : 10.51186/journals/ed.2020.10-2.e340}

Les systèmes éducatifs au Nord comme au Sud sont l'objet de multiples réformes qui visent à améliorer la qualité, la pertinence et l'efficience. La coopération internationale et la recherche apparaissent dans ce cadre comme des outils privilégiés. En effet, les résultats de la recherche en éducation sont censés fournir aux décideurs et décideuses politiques les analyses tangibles permettant de prendre des décisions éclairées. Cependant, si historiquement, le nombre de publications sur la recherche en éducation n'a jamais été aussi élevé, l'impact de cette recherche sur la pratique n'est que peu perceptible. Trop souvent axée sur des « objets » (par exemple, les learning analytics, l'apprentissage mobile, l'imprimante 3D) plutôt que sur des problèmes sociétaux (par exemple, l'inefficacité de l'éducation, l'absence d'engagement, la carence en littératie) (Reeves \& Lin, 2020), la recherche et la coopération internationale se voient confrontées aux défis de l'asymétrie. Asymétrie entre acteurs/trices, objets de recherche, questions sociétales, financements ou encore enjeux historiques et géopolitiques. De plus, la dialectique de la "protection-promotion » des données et des résultats de recherche (Hameline, 2001) se pose. Les chercheurs et chercheuses sont évidemment responsables des recherches qu'elles/ils conduisent. Cependant, elles/ils ne peuvent être responsables de la lecture des résultats de recherche faites par les décideurs et décideuses.

Ce numéro de la revue L'éducation en débats : analyse comparée est issu d'échanges qui ont eu lieu dans le cadre du projet RESET-Francophone en partenariat avec les Études doctorales en sciences de l'éducation (EDSE) de la Conférence universitaire de Suisse occidentale (CUSO). De manière critique, il cherche à s'interroger sur la formation à la méthodologie de la recherche et à son rôle dans la coopération scientifique internationale. II s'interroge notamment sur le champ des possibles quant à la science et à l'éducation ouvertes et libres - Open Science et Open Education - dans une société de la connaissance (Huisman, et al., 2012).

\section{LA FORMATION À LA MÉTHODOLOGIE DE RECHERCHE}

Les sciences de l'éducation, en tant que domaine scientifique, sont jeunes - elles ont une centaine d'années en Europe et aux États-Unis, une cinquantaine au Canada, et sont encore plus jeunes, voire inexistantes, dans les pays francophones du Sud. Or, de manière similaire au domaine de la santé, l'impact de l'éducation sur la société est important, et il est aisé de comprendre que la formation des jeunes chercheurs et jeunes chercheuses en sciences de l'éducation repose sur une responsabilité institutionnelle et sociétale. 
L'éducation, essentiellement, est à la fois un discours et une pratique. Le discours guide la pratique, et la pratique guide le discours (Bedin, et al., 2019). Les axes et les méthodes de recherche découlent de cette double articulation historique (Van der Maren, et al., 2019) avec, pour résultat, des chercheurs/euses mu-es par trois forces motrices : les théories et concepts pour continuer à mieux comprendre les sciences de l'éducation ; la formation à et par la recherche dans laquelle il s'agit de s'impliquer ; et, enfin, la contribution aux problématiques de société en éclairant notamment les politiques éducatives et les pratiques pédagogiques (Ardoino, 2000, cité par Loiola \& Kaddouri, 2016).

La formation des jeunes chercheurs/euses en éducation comprend une myriade d'objets allant des fondements du métier de chercheur et de chercheuse à la gestion de projets, en passant par la formation à et par la recherche, l'utilisation des technologies numériques, les approches méthodologiques issues des principaux paradigmes ou encore l'esprit critique.

La méthodologie représente une pièce maîtresse de la formation car elle permet de réaliser un travail académique reconnu comme tel et générant de la connaissance. Or, comment se former si les formateurs/trices ne sont elles/eux-mêmes pas au fait et/ou si elles et ils n'ont pas la capacité d'accompagner correctement ? « Les étudiants peuvent-ils apprendre ce qu'est la recherche en réalisant ce qui peut s'apparenter à une caricature de la recherche ? » (Van der Maren, et al., 2019, p. 20). Étant données les nouvelles méthodes qui ont été empruntées aux disciplines contributives, étant donnée la nécessité, pour les sciences de l'éducation, de générer des données probantes, étant données les réflexions à mener - par exemple, en termes de renouveaux épistémologiques et cognitifs -, la question du statut de la méthodologie et de sa transformation en spécialité se pose (Van der Maren, et al., 2019).

Comment donc enseigner la méthodologie de recherche en sciences de l'éducation ? Au niveau des sciences sociales, l'enseignement de la méthodologie de recherche est en quête d'une culture pédagogique partagée depuis une dizaine d'années (Earley, 2014 ; Kilburn, et al., 2014 ; Wagner, et al., 2011). C'est ainsi qu'au niveau pédagogique, trois stratégies interdépendantes se sont avérées plus efficaces que d'autres : i) rendre le processus de recherche visible en permettant aux apprenant-es de s'engager dans une recherche réelle ; ii) conduire une recherche pour s'approprier les processus, comprendre les enjeux et mieux appréhender les compétences et connaissances nécessaires pour la réaliser ; et iii) adopter une posture réflexive et critique vis-à-vis de l'expérience d'apprentissage (Lewthwaite \& Nind, 2016 ; Nind \& Lewthwaite, 2018a, 2018b).

De plus, récemment, la question de la pédagogie de l'enseignement de la méthodologie de recherche a été investiguée depuis la perspective des savoirs et connaissances ${ }^{1}$ pédagogiques et de contenu (Pedagogical Content Knowledge - PCK) de Shulman (1987). Ce travail a

\footnotetext{
${ }^{1}$ Nous traduisons knowledge par "savoirs et connaissances ». Cependant, pour alléger le texte, nous utiliserons par la suite alternativement l'un ou l'autre de ces termes.
} 
notamment permis de distinguer deux types de connaissances à développer en tant qu'enseignant-e de méthodologie de recherche en sciences sociales (Nind, 2020) : les savoirs génériques, d'une part, qui comprennent autant les aspects organisationnels ou le rythme, que les modèles d'ingénierie pédagogique et les savoirs spécifiques, d'autre part, qui comprennent des actions concrètes. Dans le détail, les savoirs génériques se retrouvent dans les sept éléments suivants :

1. Les aspects organisationnels, qui sont à considérer de deux points de vue. D'une part, en termes de granularité des acteurs/trices mobilisé-es (par exemple une classe entière, un groupe, un travail en pair ou individuel), et d'autre part, en termes de contenu et matière à sélectionner (i.e. point de départ, séquençage, fragmentation, intégration) ;

2. Le rythme qu'il s'agit d'alterner entre moments rapides et plus lents ;

3. L'apprentissage, qui se ferait par expérience, serait actif, centré sur l'apprenant-e et inclusif ;

4. Le contenu qui devrait être pertinent et attrayant (Nind, 2020) ;

5. Les théories d'apprentissage mobilisées dans la conception de l'environnement d'apprentissage (par ex. communauté de pratique (Wenger, 1998)) ;

6. Les méthodes d'enseignement convoquées durant l'enseignement-apprentissage (par ex. l'apprentissage expérientiel (Usher, 2018)) ;

7. Les modèles d'ingénierie pédagogique mobilisés dans la conception de l'environnement d'apprentissage (par ex. 4CID (Van Merriënboer \& Kirschner, 2013)).

Les savoirs spécifiques, pour leur part et indépendamment des approches qualitatives ou quantitatives, se peuvent se résumer dans les quatre actions suivantes :

1. Enseigner avec, par et sur les données de recherche authentiques - autrement dit, les données comme outil pédagogique ;

2. Considérer le processus «théorie derrière la logique - logique derrière les décisions décisions à l'origine de l'action » (Nind, 2020) ;

3. Prendre en compte les conceptions des apprenant-es (Class, et al. , 2016 ; Meyer, et al., 2005) ;

4. Prendre en compte les concepts seuils (Meyer, et al., 2010) dans l'apprentissage de la méthodologie de recherche en éducation (Class, et al., 2016 ; Kiley \& Wisker, 2009).

Pour intégrer la dimension numérique d'une formation hybride ou à distance, nous proposons de bâtir sur ces acquis de recherche et particulièrement la piste du PCK. Nous proposons d'y ajouter la dimension technologique en recourant au modèle TPACK (Technological, Pedagogical and Content Knowledge) de Mishra et Koehler (2006) car toute formation médiatisée comporte bien une dimension technologique. Essayer de la définir et de la comprendre dans ses interactions avec les autres éléments contribue à améliorer la qualité de l'environnement d'apprentissage.

Le savoir technologique (TK) est multiple et comprend autant la maîtrise de logiciels professionnels que l'accès à des jeux de données par exemple. De plus, aux intersections des 
savoirs pédagogiques et technologiques, avec le TPK (Technological and Pedagogical Knowledge), on cherche à évaluer l'impact de la technologie et des changements qu'elle pourrait apporter sur l'enseignement et l'apprentissage de la méthodologie de recherche. Comment par exemple intégrer dans son enseignement des jeux de données tels ceux du Programme international pour le suivi des acquis des élèves (PISA), du Programme d'analyse des systèmes éducatifs de la CONFEMEN (PASEC) ou de chercheurs et chercheuses lambda déposés sur Zenodo ${ }^{2}$, Olos ${ }^{3}$ ou autre Yareta ${ }^{4}$ ?

Avec le TCK (Technological and Content Knowledge), on cherche à comprendre l'impact de la technologie sur la pratique et la connaissance de l'enseignement-apprentissage de la méthodologie de recherche en l'utilisant et en développant des recherches à propos de cette utilisation. Comment est-ce que l'automatisation du processus de traitement de données impacte la recherche en sciences sociales par exemple? (Voir l'article de Class \& Akkari pour plus de détails).

\section{LA COOPÉRATION EN MATIÈRE DE RECHERCHE}

Au-delà des questions technologiques, pédagogiques et de contenu, et de l'accès, se pose la question plus large de la coopération scientifique internationale. Pour qu'il y ait coopération scientifique internationale, il faut qu'il y ait mobilité (Flanagan, 2015). En effet, les flux de savoir et de connaissance doivent pouvoir circuler pour que les " cerveaux » puissent se rencontrer, interagir et construire ensemble (Boekholt, et al., 2009). Rappelons que c'est d'ailleurs à cette fin que le web a été créé il y a plus de 30 ans - pour soutenir l'échange d'information entre chercheurs/euses internationales/aux ${ }^{5}$.

II a par ailleurs été démontré que les impacts de la coopération scientifique internationale se répercutent bénéfiquement sur quatre niveaux différents : celui de la/du chercheur/euse en tant qu'individu-e (accès aux ressources, développement d'un réseau, publications conjointes, etc.), celui de l'institution de recherche (financement, visibilité, accès à des connaissances complémentaires, etc.), celui des entreprises (par exemple, l'exploitation de nouvelles connaissances, l'accès à des technologies, etc.) et enfin celui de la politique (innovation, excellence, contribution) (Boekholt, et al., 2009 ; Gaillard \& Gaillard, 2017).

Bâtir un socle commun entre chercheurs/euses des sciences de l'éducation, à l'échelle francophone et internationale, pour permettre à un dialogue constructif de se développer pourrait être un début. C'est ainsi que guider la formation des jeunes chercheurs/euses par un référentiel de compétences (Van der Maren, et al., 2019) semble représenter un outil intéressant en vue d'améliorer la qualité, la pertinence et l'efficience pour toutes les parties prenantes. Pour ce faire, collaborer pour ouvrir la formation, notamment en termes

\footnotetext{
2 https://zenodo.org/

${ }^{3}$ https://olos.swiss/

${ }^{4}$ https://yareta.unige.ch/

${ }^{5}$ https://home.cern/science/computing/birth-web
} 
épistémologiques, pourrait constituer un premier pas. II s'agirait, dans le sens de l'éducation ouverte et libre (Blessinger \& Bliss, 2016) et de celui de la justice cognitive (Kidd, et al., 2017 ; Piron, et al. , 2016), d'ouvrir le champ des possibles épistémologiques.

En ce sens, de nouvelles méthodologies pourraient sans doute émerger pour pouvoir adresser des problèmes sociétaux plutôt que des « objets » en référence à Reeves (2020) ci-dessus. De nouveaux centres d'expertise pourraient voir le jour en fonction de problématiques contextuelles telles que, sur un plan théorique, repenser des concepts clés à la lumière de nouveaux paradigmes, ou sur un plan pratique, chercher des solutions pour intégrer, dans la société de la connaissance, tous les laissés-pour-compte des systèmes scolaires et accueillir des jeunes chercheurs et chercheuses du monde entier pour faire avancer la connaissance sur le sujet.

Les articles de ce numéro spécial sont un témoignage quant au rôle que la coopération internationale scientifique en éducation peut jouer, et se veut une contribution à la formation ouverte et libre des jeunes chercheurs et chercheuses en éducation.

\section{RÉFÉRENCES}

Blessinger, P., \& Bliss, T. J. (2016). Introduction to Open Education: Towards a Human Rights Theory. In P. Blessinger, \& T. J. Bliss (Eds.), Open Education. International Perspectives in Higher Education (p. 11-30). Cambridge: Open Book Publishers.

Boekholt, P., Edler, J., Cunningham, P., \& Flanagan, K. (2009). Drivers of International Collaboration in Research. Brussels: European commission. https://ec.europa.eu/research/iscp/pdf/publications/drivers_sti.pdf

Class, B., Schneider, D., Laroussi, M., \& Lombard, F. (2016). Enseigner la méthodologie de la recherche en technologie éducative : des conceptions aux concepts seuils. Distances et médiations des savoirs, 13, 2-17. https://doi.org/10.4000/dms.1349

Earley, M. (2014). A synthesis of the literature on research methods education. Teaching in Higher Education, 19(3), 242-253. https://doi.org/10.1080/13562517.2013.860105

Flanagan, K. (2015). International Mobility of Scientists. In D. Archibugi, \& A. Filippetti (Eds.), The Handbook of Global Science, Technology, and Innovation (p. 364-381). Chichester: John Wiley \& Sons, Ltd.

Gaillard, A.-M., \& Gaillard, J. (2017). Caractéristiques et déterminants des collaborations scientfiques entre le Maroc et l'Europe: une enquête questionnaire. In H. Bouabid, \& J. Gaillard (Eds.), La recherche scientifique au Moroc et son internationalisation (p. 201232). Saarbrücken: Éditions universitaires européennes.

Hameline, D. (2001). In memoriam Michael A. Huberman (1940-2001). Revue française de pédagogie, 137, 183-185.

Huisman, J., Adelman, C., Hsieh, C., Shams, F., \& Wilkins, S. (2012). Europe's Bologna process and its impact on global higher education. In D. K. Deardorff, H. D. Wit, \& J. D. Heyl (Eds.), The SAGE Handbook of International Higher Education (p. 81-100). Thousand Oaks, CA: SAGE Publications, Inc. https://doi.org/10.4135/9781452218397

Kidd, I., Medina, J., \& Pohlhaus, G. (Eds.). (2017). The Routledge Handbook of Epistemic Injustice. New York, NY: Routledge. 
Kilburn, D., Nind, M., \& Wiles, R. (2014). Learning as Researchers and Teachers: The Development of a Pedagogical Culture for Social Science Research Methods? British Journal of Educational Studies, 62(2), 191-207. https://doi.org/10.1080/00071005.2014.918576

Kiley, M., \& Wisker, G. (2009). Threshold concepts in research education and evidence of threshold crossing. Higher Education Research \& Development, 28(4), 431-441. https://doi.org/10.1080/07294360903067930

Lewthwaite, S., \& Nind, M. (2016). Teaching Research Methods in the Social Sciences: Expert Perspectives on Pedagogy and Practice. British Journal of Educational Studies in Continuing Education, 64(4), 413-430. https://doi.org/10.1080/00071005.2016.1197882

Loiola, F., \& Kaddouri, M. (2016). La formation à la recherche aux cycles supérieurs : finalités, usages et enjeux. Le cas des sciences de l'éducation. TransFormations, 15-16, 1-6.

Meyer, C., Shanahan, J., \& Laugksch, R. (2005). Students' Conceptions of Research: A qualitative and quantitative analysis. Scandinavian Journal of Educational, 49(3), 225244.

Meyer, J., Land, R., \& Baillie, C. (2010). Threshold Concepts and Transformational Learning (vol. 42). Rotterdam: Sense Publishers.

Mishra, P., \& Koehler, M. (2006). Technological Pedagogical Content Knowledge: A Framework for Teacher Knowledge. Teachers College Record, 108(6), 1017-1054.

Nind, M. (2020). A new application for the concept of pedagogical content knowledge: teaching advanced social science research methods. Oxford Review of Education, 46(2), 185201. https://doi.org/10.1080/03054985.2019.1644996

Nind, M., \& Lewthwaite, S. (2018a). Hard to teach: inclusive pedagogy in social science research methods education. International Journal of Inclusive Education, 22(1), 74-88. https://doi.org/10.1080/13603116.2017.1355413

Nind, M., \& Lewthwaite, S. (2018b). Methods that teach: developing pedagogic research methods, developing pedagogy. International Journal of Research \& Method in Education and Information Technologies, 41(4), 398-410. https://doi.org/10.1080/1743727X.2018.1427057

Piron, F., Regulus, S., \& Djiboune Madiba, M. S. (2016). Justice cognitive, libre accès et savoirs locaux. Pour une science ouverte juste, au service du développement local durable. Québec, QC : Science et bien commun.

Reeves, T. C., \& Lin, L. (2020). The research we have is not the research we need. Educational Technology Research and Development, 68(4), 1991-2001. https://doi.org/10.1007/s11423-020-09811-3

Shulman, L. (1987). Knowledge and teaching: Foundations of the new reform. Harvard Educational Review, 57(1), 1-22.

Usher, R. (2018). Experiential learning. In K. Illeris (Ed.), Contemporary theories of learning $\left(2^{e}\right.$ éd., p. 189-203). London: Routledge.

Van der Maren, J.-M., Brodeur, M., Gervais, F., Gilles, J.-L., \& Voz, G. (2019). Référentiel pour la formation des chercheuses et des chercheurs francophones en éducation. Québec, QC: ADEREQ. http://hdl.handle.net/20.500.12162/2746

Van Merriënboer, J., \& Kirschner, A. (2013). Ten steps to complex learning: a systematic approach to four-component instructional design ( $2^{e}$ éd.). Vancouver, BC: Routledge.

Wagner, C., Garner, M., \& Kawulich, B. (2011). The state of the art of teaching research methods in the social sciences: towards a pedagogical culture. Studies in Higher Education, 36(1), 75-88. 
Wenger, E. (1998). Communities of Practice: Learning, Meaning, and Identity. Cambridge: Cambridge University Press.

Open Access Publications - Bibliothèque de l'Université de Genève Creative Commons Licence 4.0

(c) (1) 